\title{
MEASUREMENTS OF SMALL ION MOBILITY SPECTRUM WITH MULTI-ELECTRODES GERDIEN CONDENSER
}

\author{
K. Suzuki, M. Iritani, and T. Mitsukuchi
}

Research Institute of the Magnetosphere, Chubu Institute of Technology Kasugai, 487

Abstract. The characteristics of our data on small ion mobility spectrum are described and the instrumentation problems are considered.

1. Introduction

Small ion mobility spectrum is an important factor to influence the atmospheric electric conductivity. The measurements were made with the multielectrode Gerdien condenser, which can reduce the time required for measuring the whole range of small ion mobility. This paper reports the results of recent measurements.

2. Principles of the measurement

The present instrument is pictured in Fig. 1.

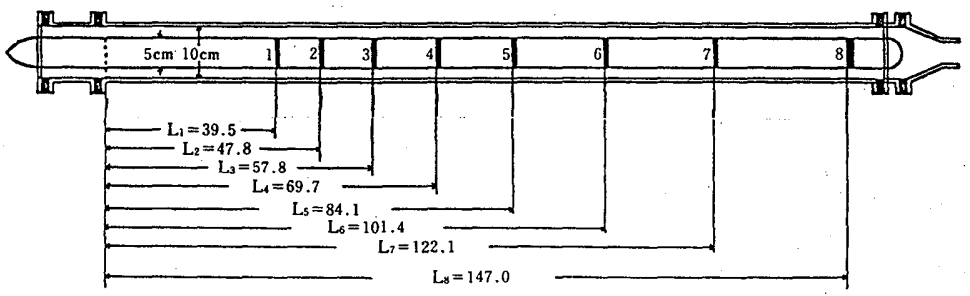

Fig. 1. Schematic diagram of multi-electrode condenser.

The formula for a mobility spectrum density $f(k)$ and the present experimental conditions are as follows,

$$
\begin{aligned}
& \int_{k_{j}}^{k_{j-1}} f(k) d k=\left[1 / e \phi_{0}\right] \partial\left(k_{j} I_{j}\right) / \partial k_{j} \quad(j=2,3,4,5,6,7,8) \\
& k_{j}=\phi_{0} \ln \left(r_{o} / r_{i}\right) /\left[2 \pi L_{j} V\right]
\end{aligned}
$$

where $e$ : elementary electric charge, $\phi_{0}$ (the flux of air flow) $=158[1 / \mathrm{m}], I_{j}$ : the electric current of ions captured by the electrode $j, r_{i}$ (the inner radius $)=2.5[\mathrm{~cm}], r_{O}($ the outer $\operatorname{radius})=5[\mathrm{~cm}], L_{j}$ : the total length of all electrodes from the $1-s t$ to the $j-$ th one, $V$ : the applied voltage, $k_{j-1} / k_{j}$ (the ratio of critical mobilities) $=1.2$. Equation (1) is based on the two assumptions of $k_{j-1} / k_{j}=$ const. and $\phi_{0}=$ const., where $k_{j}$ can be considered to be the independent variable of the function $I_{j}\left(k_{j}\right)$.

We tried to change the value of $k_{j}$ by changing $L_{j}$ and $V$. Seven electrodes $(j=2,3, \cdots, 8)$ shown in Fig. 1 provide the values $I_{j}$ for seven different values of $k_{j}$ simultaneously; each electrode is connected with one electrometer. Moreover, we changed the applied voltage for each polarity in three steps. Owing to the multi-electrode condenser, the time required for obtaining the $7 \times 3=21$ observed points at $\left[k_{j}-k_{j} I_{j} / e \phi_{0}\right]$ curve is remarkably reduced as compared with the usual two electrode type. The results of the first research of multi-electrode Gerdien condenser were presented by Brownlee(1975). 
3. Results

The mobility spectrum in Eq. (1) is calculated from the gradient of the characteristic curve of $\left[k_{j}-k_{j} j_{j} / e \phi_{0}\right]$. An example of this curve for positive ions is shown in Fig. 2 .

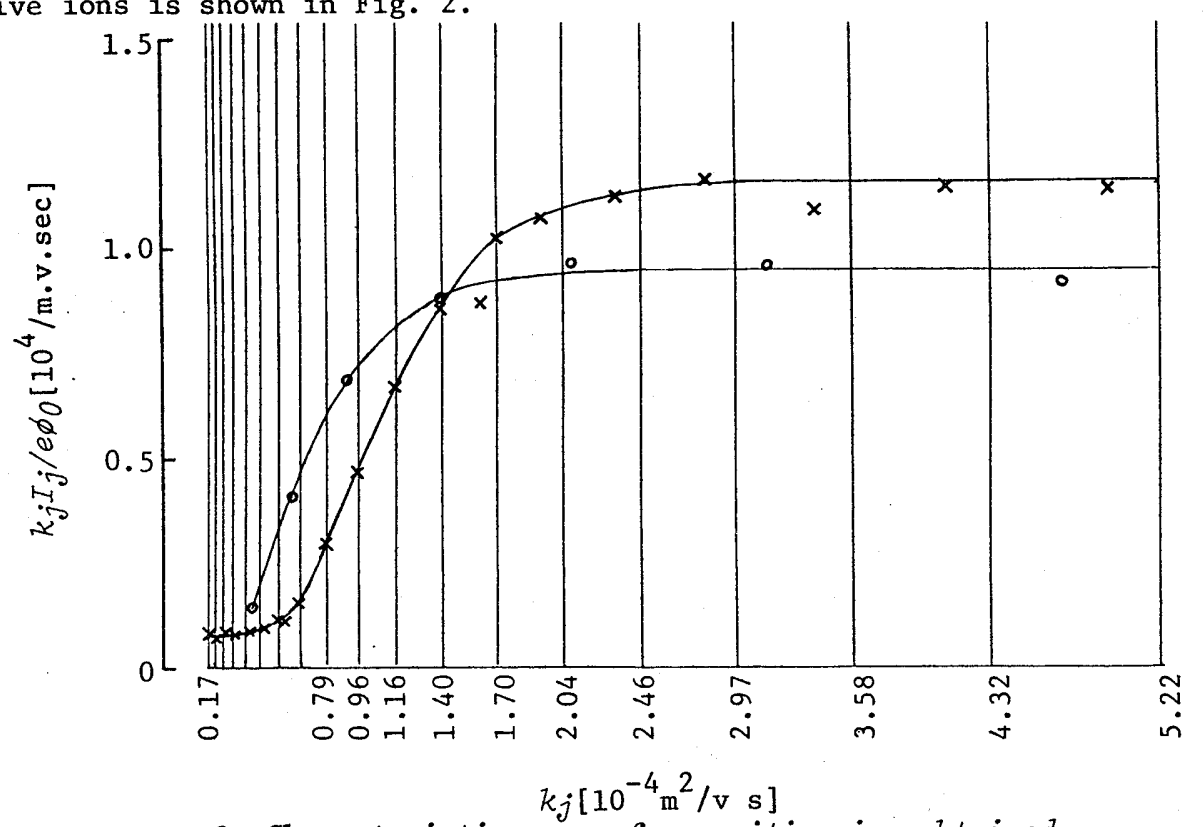

The 21 observed points

Fig. 2. Characteristic curve for positive ion obtained during from 7:25 to 7:53, May 30, 1981.

(denoted by sign $x$ ) do not

always form a smooth curve due to the inclusion of several kinds of errors. However, these points sufficiently allow us to draw a fully fitting smooth curve. We added here the similar data for the case of the usual two electrode condenser (denoted by sign o) just for reference; it is as a matter of fact unappropriate to add them in the same figure, because the width of one mobility range interval for a spectrum in the case of the two electrode condenser $\left(k_{1} / k_{2}=1.5\right)$ is different from that in the case of the multi-electrode type.

Shown in Fig. 3 is the mobility spectrum with 18 intervals,

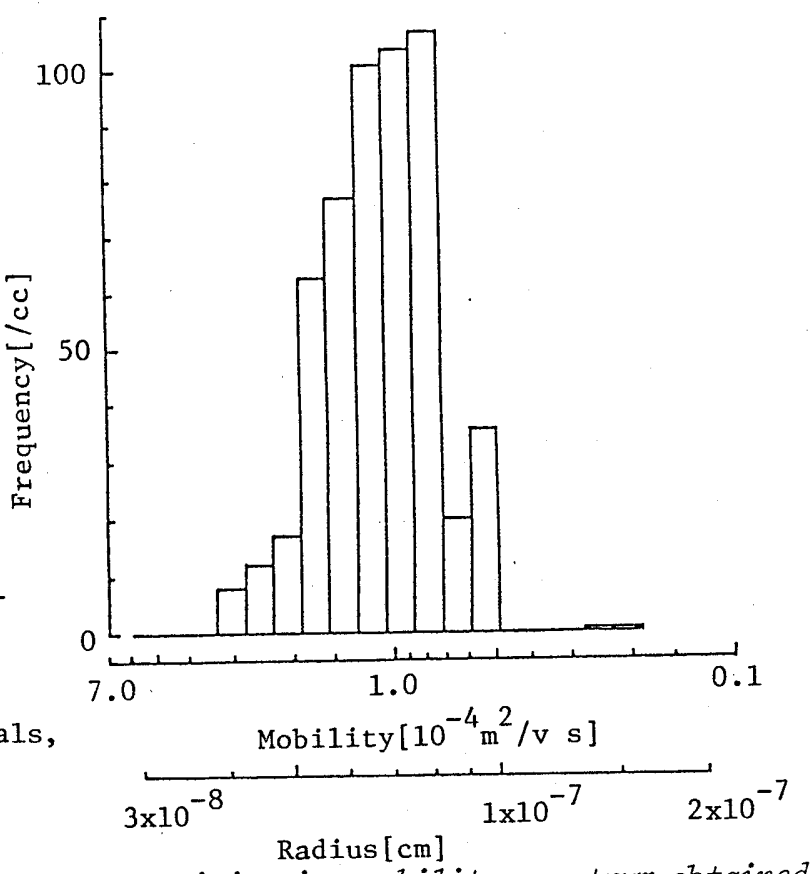

Fig. 3. Positive ion mobility spectrum obtained during from $7: 25$ to $7: 53$, May 30, 1981. 
K. Suzuki, M. Iritani, and T. Mitsukuchi

for positive ions obtained from the fitting curve corresponding to Fig. 2. We show in Fig. 4 the spectrum for negative ions obtained at the same time as Fig. 3.

Let us investigate the following three parameters. (i) Conductivity: the electric conductivity is not only directly obtained by

$$
\lambda_{o b}=I_{2} /\left[\phi_{0}\left(1 / k_{2}-1 / k_{1}\right)\right]
$$

but also can be calculated by

$$
\lambda_{\text {cal }}=e \sum_{j=2}^{18} \sqrt{k_{j} k_{j-1}} n_{j}
$$

where $n_{j}=\int k_{j-1}^{k_{j}} f(k) d k$

(ii) Mode: the value of the mode of the mobility spectrum was calculated by

$$
k_{\text {mode }}=k_{j 0-1}^{(1+d) / 2} k_{j 0}^{(1-d) / 2}
$$

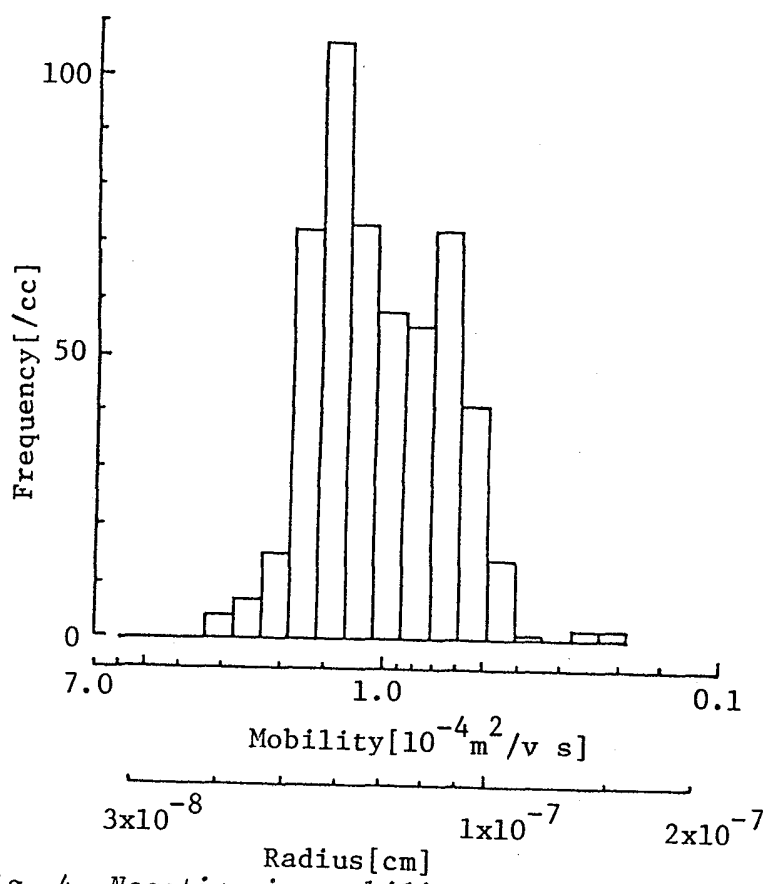

ig. 4. Negative ion mobility spectrum obtained during from $7: 25$ to $7: 53$, May 30, 1981 .

where $d=\left(n_{j_{0}-1}-n_{j_{0}+1}\right) / n_{j_{0}}$, and $n_{j_{0}}$ denotes the $j_{0}$ th spectrum density, which corresponds to the peak of the mobility spectrum (illustrated in Fig. 5). This formula was obtained by considering the neighboring spertrum density.

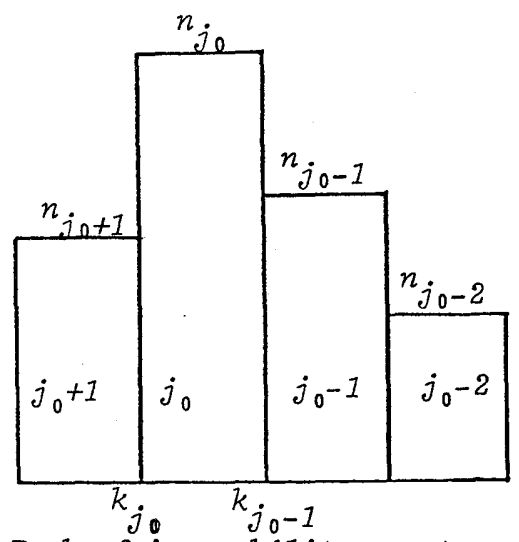

Table 1. Mode of small ion mobility spectrum

\begin{tabular}{|l|l|l|}
\hline \multicolumn{2}{|c|}{$\left[\mathrm{x} 10^{-4} \mathrm{~m}^{2} / \mathrm{v}\right.$ s $]$} \\
\hline Misaki's Koenji (1965) & positive & negative \\
Ogasawara (1972) & $1.257+0.006$ & $1.560+0.007$ \\
main island coast (1973) & $1.028+0.021$ & $1.304+0.037$ \\
$1.023+0.007$ & $1.239 \pm 0.017$ \\
Israel (1957) & 1.37 & 1.89 \\
Hoppel and Kraakevik (1965) & 1.15 & 1.24 \\
Our study (1981) & 1.11 & 1.40 \\
\hline Average of small ion mobility & 1.15 & 1.21 \\
\hline
\end{tabular}


(iii) Average: the average value of mobility is defined by $k_{a v}=\sum_{j} \sqrt{k_{j} k_{j-1}} n_{j} / \sum_{j} n_{j}$

For example, $\lambda_{o b}=10^{j} .9\left[\times 10^{4} \mathrm{~m}^{2} / \mathrm{v} \mathrm{s}\right]$ and $\lambda_{c a l}=10.1$ for Fig. 3 , and $\lambda_{o b}=10.1$ and $\lambda_{c a l}=9.5$ for Fig. 4. The values of the mode and the average values of mobility of our study are presented in Table 1 together with the conventional ones (Ikegami et al. 1976). Our study is based on 17 data of the ion mobility spectrum obtained during from 5:05 to 13:01, May 30, 1981 .

One scanning time to measure the whole range of one spectrum is 12 minutes for our multi-electrode condenser and 24 minutes for the usual two electrode condenser of our own.

4. Concluding remarks and discussion

Preliminary results are as follows; more measurement is required.

(i) The value of the mode and the average value of mobility of positive small ions are smaller than those of negative ions.

(ii) The total number of positive small ions is $5-10 \%$ larger than that of negative small ions.

(iii) The calculated value of conductivity $\lambda_{c a l}$ was in perfect agreement with the observed one $\lambda_{o b}$.

(iv) The values of conductivity obtained with our multi-electrode condenser are about twice as high as the common values observed on the ground. The authors believe this is due to the following situation. Our multi-electrode condenser was set up in a reinforced concrete room, and before entering the measurement system the air sample was kept in a container for a few minutes and suffered radio activity from the wall of the room more than the ambient air. Shown in Fig. 6 are the data of conductivity for our multi-electrode condenser set up in the room and for the usual two electrode condenser of our own which was set up outside the room. When we brought the latter inside the room, both instruments provided us with almost the same data. Thus, the location of the instrument turned out to be a very important factor influencing the value of electric conductivity of our ambient air.

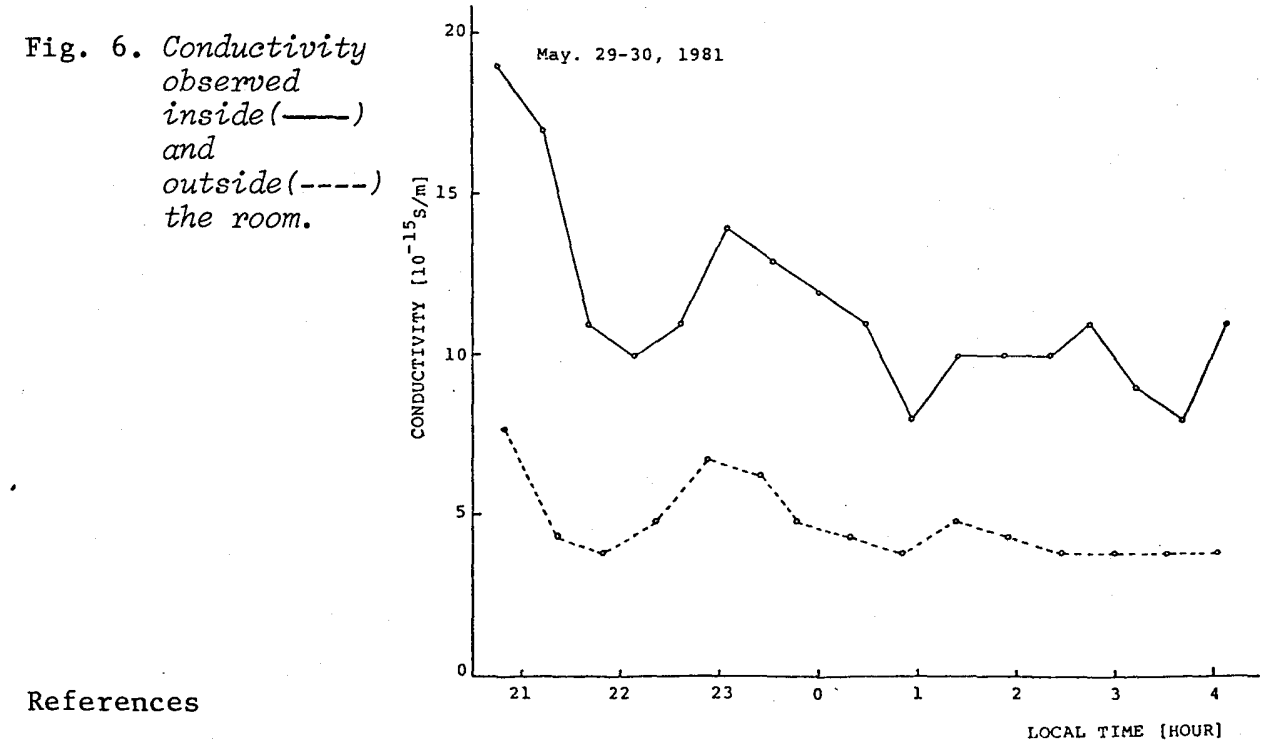

Brownlee, J. N., A new approach to atmospheric-ion mobility spectrometry, J. Atmos. and Terr. Phys., 37, 1139-1144, 1975.

Ikegami, M., M. Misaki, and I. Kanazawa, Sma11 ion mobility on the sea, Proc. Soc. Atmos. Electr. Japan, 15, 110-115, 1976 (in Japanese).

(Received September 28, 1981; revised March I, 1982;

- 4 - accepted March 27, 1982) 